\title{
The effect of needle tip position on the analgesic efficacy of pulsed radiofrequency treatment in patients with chronic lumbar radicular pain: a retrospective observational study
}

\author{
Won-Joong Kim ${ }^{1}$, Hahck Soo Park ${ }^{2}$, and Min Ki Park ${ }^{1}$ \\ 'Department of Anesthesiology and Pain Medicine, Ewha Womans University Mokdong Hospital, School of Medicine, Ewha Womans University, \\ Seoul, Korea \\ ${ }^{2}$ Department of Anesthesiology and Pain Medicine, Ewha Womans University Seoul Hospital, School of Medicine, Ewha Womans University, Seoul, \\ Korea
}

Received March 12, 2019

Revised August 15, 2019

Accepted August 18, 2019

\section{Correspondence}

Won-Joong Kim

Department of Anesthesiology and Pain Medicine, Ewha Womans University

Mokdong Hospital, School of Medicine,

Ewha Womans University, 1071

Anyangcheon-ro, Yangcheon-gu, Seoul

07985, Korea

Tel: +82-2-2650-2689

Fax: +82-2-2655-2924

E-mail: ickypoo@naver.com

Key Words

Analgesics

Ganglia, Spinal

Low Back Pain

Lumbosacral Region

Needles

Pulsed Radiofrequency Treatment

Radiculopathy

Spinal Nerve Roots
Background: Pulsed radiofrequency (PRF) is a treatment modality that alleviates radicular pain by intermittently applying high-frequency currents adjacent to the dorsal root ganglion. There has been no comparative study on analgesic effect according to the position of the needle tip in PRF treatment. The objective of this study is to evaluate the clinical outcomes of PRF according to the needle tip position.

Methods: Patients were classified into 2 groups (group IP [group inside of pedicle] and group OP [group outside of pedicle]) based on needle tip position in the anteroposterior view of fluoroscopy. In the anteroposterior view, the needle tip was advanced medially further than the lateral aspect of the corresponding pedicle in group IP; however, in group OP, the needle tip was not advanced. The treatment outcomes and pain scores were evaluated at 4,8 , and 12 weeks after applying PRF. Results: At 4, 8, and 12 weeks, there were no significant differences between the successful response rate and numerical rating scale score ratio.

Conclusions: The analgesic efficacy of PRF treatment did not differ with the needle tip position.

\section{INTRODUCTION}

Lumbar radicular syndrome is a disease that involves radiating pain in one or more dermatomes, and may be accompanied by chronic nerve irritation and dysfunction. Lumbar radicular syndrome is a highly prevalent disease, but a challenging clinical problem. Interventional procedures such as epidural steroid injection (ESI) may be considered if conservative treatments, such as physical therapy or medication are initially tried and are not effec- tive.

Pulsed radiofrequency (PRF) is a treatment modality that alleviates radicular pain by intermittently applying high-frequency currents adjacent to the dorsal root ganglion (DRG) [1,2]. Although the mechanism of PRF is not fully understood, the electric field reversibly blocks transmission without destroying the small unmyelinated fibers, and the large fibers continue to be protected by the myelin sheath [3].

The DRG plays an important role in low back pain and (c) This is an open-access article distributed under the terms of the Creative Commons Attribution Non-Commercial License (http://creativecommons.org/licenses/by-nc/4.0/), which permits unrestricted non-commercial use, distribution, and reproduction in any medium, provided the original work is properly cited.

(c) The Korean Pain Society, 2019
Author contributions: Won-Joong Kim: Study conception; Hahck Soo Park: Investigation; Min Ki Park: Writing/manuscript preparation. 


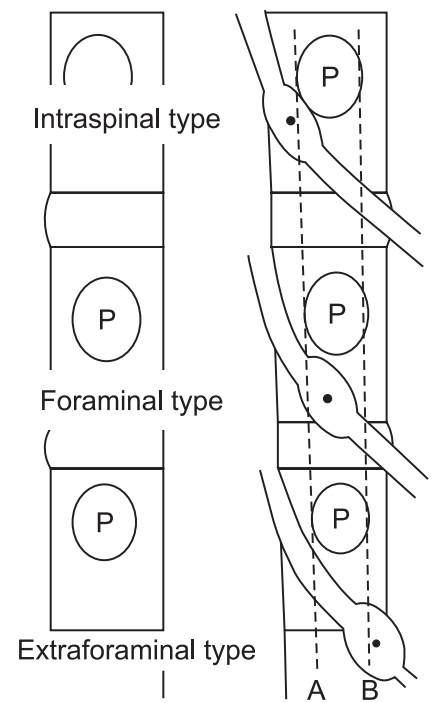

Fig. 1. Determination of the dorsal root ganglion (DRG) position. A and $B$ are lines connecting the medial and lateral borders of the pedicles, respectively. If the midpoint of the DRG lies proximal to $A$, it is intraspinal type. If it is between $A$ and $B$, it is foraminal type. If it is distal to $B$, it is extraforaminal type.

radiating pain [4]. The DRG is very sensitive to mechanical compression, and is closely related to abnormal sensation and radiating pain [5]. Radiologically, the location of the DRG is divided into 3 types-the intraspinal, foraminal, and extraforaminal regions (Fig. 1) - and most DRG neurons are of the foraminal type [6]. This position corresponds to the dorsal-cranial quadrant of the intervertebral foramen on the lateral view in fluoroscopy, and the middle of the pedicle column on the anteroposterior (AP) view. However, if the arthritic degenerative changes and foraminal stenosis are severe, positioning the needle to target the DRG on fluoroscopy may be difficult [7]. Accordingly, needle tips can be placed laterally on the side of the corresponding pedicle in the AP view.

To our knowledge, there has been no comparative study on how analgesic effects correspond to the position of the needle tip in PRF treatment. The purpose of this study was to evaluate the clinical effects of PRF according to the needle tip position.

\section{MATERIALS AND METHODS}

\section{Patient population}

This study was approved by the Institutional Review Board (IRB) of Ewha Womans University Hospital (EUMC 201811-006). The patient's information and records were anonymized, and then the IRB waived the written informed consent requirement. We collected clinical data through a hospital database and analyzed records for all patients with lumbar radiating pain who underwent PRF adjacent to the DRG between March 2016 and December 2017.

Inclusion criteria were as follows: (1) pain duration $>3$ months; (2) unilateral radiating pain, suggesting involvement of the lumbar spinal nerves (L4 and L5) as well as affected nerve roots consistent with magnetic resonance imaging (MRI) findings; (3) being 20 years of age or older; (4) a score of 4 or more on an 11-point numerical rating scale (NRS) for pain scores after conservative treatment, including oral medication, physical therapy, or ESI; and (6) foraminal type on spine MRI (Fig. 1) [6].

Exclusion criteria were as follows: (1) absence of spinal MRI; (2) inadequate management of coexisting psychiatric diagnoses; (3) history of cancer, lumbar fracture, systemic disease, or connective tissue diseases; (4) no 3-month follow-up data; (5) pregnancy, coagulation disorders, systemic infection, fever, or local infection at the puncture site; (6) presence of a cardiac pacemaker or spinal cord stimulator; and (7) PRF treatment of the DRG in the last year; and (8) position of the needle tip in the caudal portion of the intervertebral foramen on the lateral view in fluoroscopy $[8,9]$.

Patient characteristics assessed included age, sex, weight, height, comorbidities (such as hypertension and diabetes mellitus), and pain duration and location. The medication quantification scale III (MQS) was used to quantify changes in analgesics [10]. We also analyzed clinical factors such as treatment level, spinal surgery history, MRI findings, the presence of disc herniation, target level foraminal stenosis, and central stenosis. We also analyzed the number of ESIs performed at the pain clinic for 3 months before PRF treatment.

\section{PRF procedure}

For PRF, the patient was placed in a prone position, and a pillow was placed under the lower abdomen. Disinfection was performed at the procedure site, and a sterile drape was put in place. Patients received at least 2 ESIs in the 3 months preceding PRF treatment, and a diagnostic root block was not performed immediately before PRF treatment. The entire course of the procedure was performed under fluoroscopic guidance. After local anesthesia with $1 \%$ lidocaine was applied to the skin, the RF needle (22 G, $10 \mathrm{~cm}$, curved, with $10-\mathrm{mm}$ active tip) was inserted into the target neuroforamen. The target point was the dorsalcranial quadrant of the intervertebral foramen in the lateral view, and midway into the pedicle column in the AP view. When the RF needle was close to the target position, the stylet of the RF needle was removed, and the RF probe was inserted. The final position of the RF probe was determined with sensory stimulation $(50 \mathrm{~Hz})$, or when the pa- 
tient felt a tingling sensation at a voltage below $0.5 \mathrm{~V}$. If the threshold value exceeded $0.5 \mathrm{~V}$, the needle was carefully advanced until the patient felt sensory stimulation. Pulsed current $(20 \mathrm{msec}, 2 \mathrm{~Hz})$ was then applied 3 times for 120 seconds each time, with a $45-\mathrm{V}$ output and short intervals between treatments. During this procedure, the temperature at the tip of the electrode was not supposed to exceed $42^{\circ} \mathrm{C}$.

\section{Clinical data}

The success of the procedure was defined as a decrease of $50 \%$ or more in the NRS score without increasing analgesic consumption [11,12]. All other responses were deemed negative responses. The patients were classified into 2 groups, group IP (group inside of pedicle) and group OP (group outside of pedicle), according to the position of the needle tip in the AP view. The position of the needle tip was analyzed by two physicians who were not involved in the procedure: one was an experienced pain physician, and the other was a radiologist. Using an AP view, the needle tip was advanced further inward than the lateral aspect of the corresponding pedicle in group IP. However, the tip of the needle did not move forward in group OP (Fig. 2). We evaluated the success rate of treatment and NRS scores at 4 , 8 , and 12 weeks after PRF, and reviewed all complications within 3 months after the procedure.

\section{Statistical analysis}

Continuous variables are demonstrated as mean \pm standard deviation or medians (interquartile ranges), and categorical variables are displayed as numbers (percentages). Demographic and clinical data were compared between the 2 groups by $t$-test, chi-square test, or Mann-Whitney $U$-testing. $P$ values less than 0.05 were considered statistically significant. All statistical analyses were performed using PASW ver. 18.0 (IBM Corp., Armonk, NY).

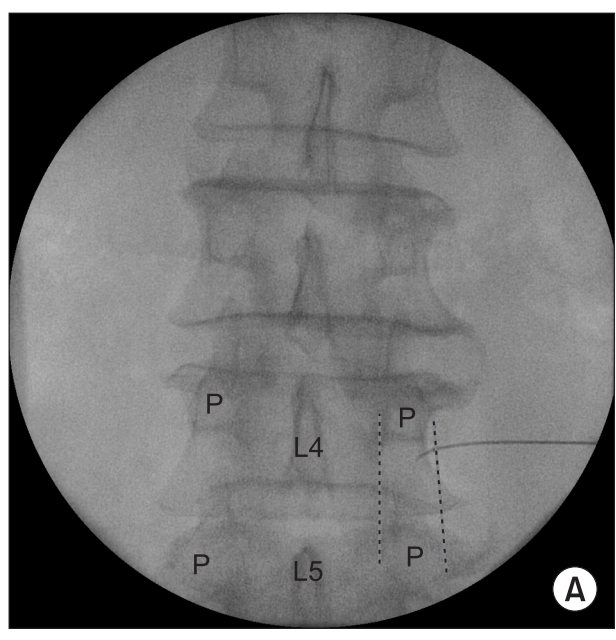

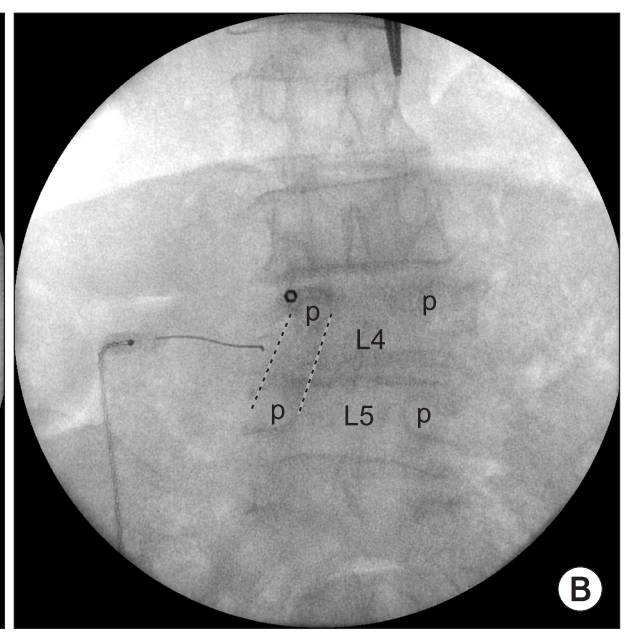

B
Fig. 2. Position of the needle tip. (A) The needle tip was advanced medially further than the lateral aspect of the corresponding pedicle. (B) The needle tip was in the lateral aspect of the corresponding pedicle. P: pedicle.

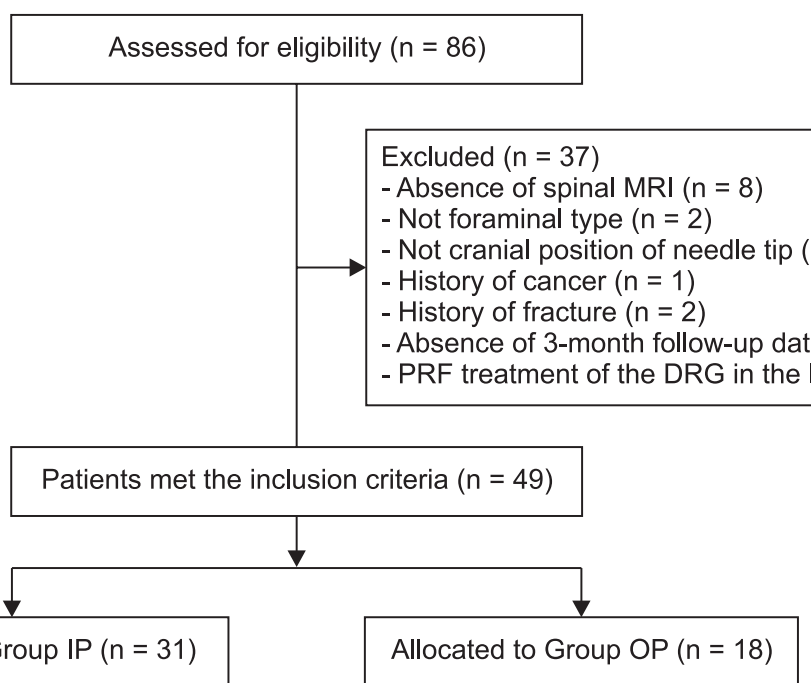

Fig. 3. Flow diagram of the patient inclusion process. MRI: magnetic resonance imaging, PRF: pulsed radiofrequency, DRG: dorsal root ganglion, Group IP: group inside of pedicle, Group OP: group outside of pedicle. 
Table 1. Demographic Data

\begin{tabular}{|c|c|c|c|}
\hline Variable & Group IP $(n=31)$ & Group OP $(n=18)$ & $P$ value \\
\hline Age (yr) & $71 \pm 11$ & $71 \pm 12$ & 0.93 \\
\hline Sex (male/female) & $19(61.3) / 12(38.7)$ & $7(38.9) / 11(61.1)$ & 0.13 \\
\hline Height (cm) & $162 \pm 8$ & $159 \pm 10$ & 0.13 \\
\hline Weight (kg) & $67 \pm 10$ & $64 \pm 12$ & 0.36 \\
\hline Hypertension & $18(58.1)$ & $12(66.6)$ & 0.55 \\
\hline Diabetes mellitus & $5(16.1)$ & $2(11.1)$ & 0.63 \\
\hline \multicolumn{4}{|l|}{ MQS } \\
\hline Baseline & $9.3(6.3)$ & $10.2(4.6)$ & 0.98 \\
\hline 4 wk after PRF & $5.6(5.8)$ & $10.2(5.3)$ & 0.95 \\
\hline 8 wk after PRF & $7.9(6.4)$ & $10.2(5.3)$ & 0.99 \\
\hline 12 wk after PRF & $7.9(8.5)$ & $10.2(5.3)$ & 0.86 \\
\hline \multicolumn{4}{|l|}{ Pain duration (mo) } \\
\hline $3-6 / 6-12 />12$ & $9(29.0) / 4(12.9) / 18$ (58.1) & $2(11.1) / 5(27.8) / 11(61.1)$ & 0.22 \\
\hline Location of pain (left/right) & $12(38.7) / 19(61.3)$ & $11(61.1) / 7(38.9)$ & 0.13 \\
\hline Levels treated (L4/L5) & $11(35.5) / 20(64.5)$ & $9(50.0) / 9(50.0)$ & 0.32 \\
\hline Failed back surgery syndrome & $4(12.9)$ & $3(16.7)$ & 0.72 \\
\hline Central stenosis & $23(74.2)$ & $15(83.3)$ & 0.46 \\
\hline Mild/Moderate/Severe & $13(56.5) / 8(34.8) / 2(8.7)$ & $5(33.3) / 7(46.7) / 3(20.0)$ & 0.33 \\
\hline Multiple central stenosis & $8(25.8)$ & $5(27.8)$ & 0.88 \\
\hline Foraminal stenosis & $10(32.3)$ & $8(44.4)$ & 0.39 \\
\hline Herniated intervertebral disc & $21(67.7)$ & $13(72.2)$ & 0.74 \\
\hline No. of previous ESI & $2(2)$ & $2(2)$ & 0.90 \\
\hline
\end{tabular}

Values are presented as the mean \pm standard deviation, median (interquartile range), or number (\%).

Group IP: group inside of pedicle, Group OP: group outside of pedicle, MQS: medication quantification scale, PRF: pulsed radiofrequency, ESI: epidural steroid injection.

Table 2. Successful Outcome

\begin{tabular}{lcrc}
\hline \multicolumn{1}{c}{ Variable } & $\begin{array}{r}\text { Group IP } \\
(\mathrm{n}=31)\end{array}$ & $\begin{array}{r}\text { Group OP } \\
(\mathrm{n}=18)\end{array}$ & $P$ value \\
\hline Success at 4 wk after PRF & $17(54.8)$ & $11(61.1)$ & 0.67 \\
Success at 8 wk after PRF & $15(48.4)$ & $11(61.1)$ & 0.39 \\
Success at 12 wk after PRF & $12(38.7)$ & $8(44.4)$ & 0.69 \\
\hline
\end{tabular}

Values are presented as number (\%).

Group IP: group inside of pedicle, Group OP: group outside of pedicle, PRF: pulsed radiofrequency.

\section{RESULTS}

We reviewed the medical records of 86 consecutive patients who received PRF during the study period. Of these patients, 49 patients were included in our study, and 37 patients were excluded (Fig. 3).

Patient demographics and characteristics are shown in Table 1. There were no significant differences between group IP and group OP in age, sex, height, weight, pain duration, or other past medical histories. There was no significant change in the MQS during the follow-up period, and there was no difference between the 2 groups. The presence of central stenosis, foraminal stenosis, or disc herniation on MRI did not differ, and the grade of central

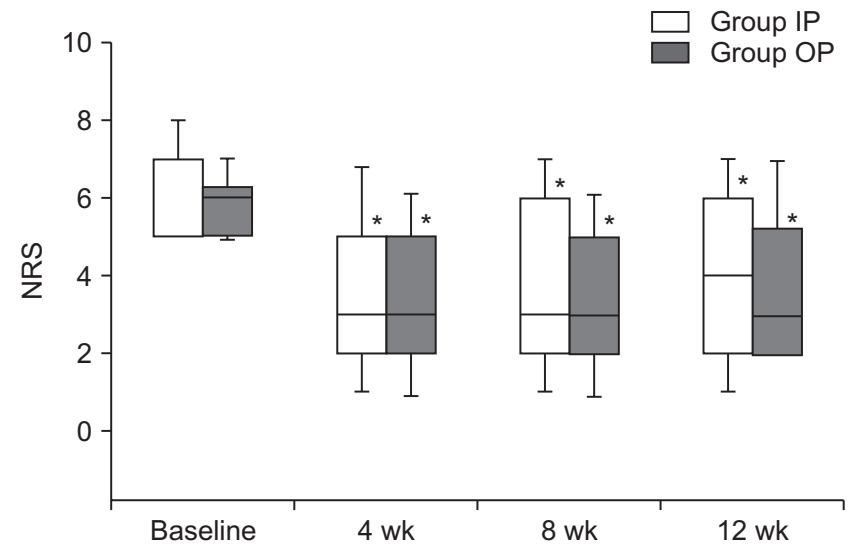

Fig. 4. Numerical rating scale (NRS). Group IP: group inside of pedicle, Group OP: group outside of pedicle. $* P<0.05$ relative to baseline.

stenosis also did not exhibit a statistically significant difference between the 2 groups. In both groups, the median number of previous ESIs before PRF was 2, and there was no difference in injection target levels or injection sites.

Table 2 shows the comparison of the success rates (based on predefined success criteria) for the 2 groups. Overall success rates were $57 \%, 53 \%$, and $40 \%$ at 4 weeks, 8 weeks, and 12 weeks, respectively (not shown), but there was no significant difference in the percentage of successful re- 
sponses between the 2 groups. There was no significant difference in baseline NRS scores. Both groups showed significant improvement in NRS scores at 4, 8, and 12 weeks after treatment, but no statistically significant differences were observed between the 2 groups (Fig. 4).

\section{DISCUSSION}

Our results demonstrated that there was no significant difference in the percentage of successful response rates and pain scores based on position of the needle tip.

In an animal study, acute lumbar nerve root compression did not cause repetitive firing for more than a few seconds, but produced an instantaneous discharge in $\mathrm{A} \delta$ and C fibers. However, acute compression of the DRG caused a long-term repeated firing (5-25 $\mathrm{min}$ ); however, this discharge occurred in both rapidly-conducting and slowlyconducting fibers ( $\mathrm{A} \beta, \mathrm{A} \delta$, and $\mathrm{C}$ fibers) [13]. PRF was found to block the signal only in unmyelinated $\mathrm{C}$ fibers, leaving myelinated $A \delta$ fibers functioning and able to transmit pain signals [14]. Das et al. [12] reported that chronic radicular pain is a centrally mediated neuroimmune phenomenon, and the mechanism of action of DRG PRF is immunomodulatory: $\mathrm{CD}^{2} 6^{+}, \mathrm{CD}^{-}$, natural killer (NK) cell frequencies, and interferon-c (IFN-c) levels decreased, while CD8 ${ }^{+} \mathrm{T}$ cell frequencies and interleukin-6 (IL-6) levels increased in treatment responders. There was an inverse correlation between IL-17 and pain severity scores after treatment.

Some physicians have suggested that PRF of the DRG is not a validated technique, and is instead a sham procedure for the treatment of lumbar radicular pain syndromes [15]; other physicians have reported that PRF is a useful intervention. Chao et al. [16] reported that at 3 months after lumbar PRF, $44.83 \%$ of patients experienced pain reduction of more than $50 \%$. In another study, $41 \%$ of patients had pain relief of more than $50 \%$ at 6 months after PRF [1]. In a study by Simopoulos et al. [17], the pain intensity of $70 \%$ of the patients was successfully reduced at 2 months after PRF treatment. Therefore, whether PRF is efficacious in the treatment of lumbar radicular pain should be considered carefully.

Radiologically, DRG positions can be classified into 3 types: intraspinal, foraminal, and extraforaminal. These types depend on the relationship between the midpoint of the DRG and the borderline of the DRG. In the intraspinal type, the midpoint of the DRG is located inside the pedicle, and in the extraforaminal type, the midpoint of the DRG is located outside the pedicle. In the foraminal type, the midpoint of the DRG is located between the medial and lateral margins of the pedicle (Fig. 1) [6]. Most DRG neurons in the upper lumbar spine (L1-3) are of the foraminal or extraforaminal type, while the DRG neurons of the lower lumbar spine (L4-5) have little extraforaminal type DRGs $[6,18]$.

Generally, a spinal nerve root block (SNRB) is performed for diagnostic purposes before DRG PRF. If there is pain reduction after SNRB, the corresponding DRG is thought to have a pathological cause. However, PRF can be performed even when there is no effect on conservative treatments, such as SNRB, in clinical practice. Furthermore, PRF may be performed to extend the effect when the SNRB has a short analgesic effect [11].

This retrospective, observational study had some limitations. First, the widths and lengths of DRG neurons are variable [6], and the asymmetry of the location of DRG neurons may be caused by disc herniation or degenerative hypertrophic facets in symptomatic patients $[19,20]$. Second, the study was conducted in a single clinical setting, and the sample size was small. Third, we did not collect information on disability and quality of life parameters other than pain relief. Fourth, we did not perform subgroup analysis on different diagnoses, such as multilevel central canal stenosis, herniated intervertebral discs, and failed back surgery syndrome; however, there was no significant difference in the diagnosis percentage between the 2 groups. Fifth, a retrospective observational study has inherent limitations. But we believe that a prospective study in which the needle tip is positioned in the lateral aspect of the pedicle intentionally would be an ethical issue, and thus, a retrospective study was best. Our results provide a basis for future prospective studies on the clinical effects of PRF according to the position of the needle tip.

In conclusion, the analgesic efficacy of PRF treatment was not statistically different based on the position of the needle tip. A larger, controlled, prospective study should be conducted to investigate the effect of needle tip position on the analgesic effects of PRF.

\section{ACKNOWLEDGMENTS}

We thank Lee HA for statistical consultation. We thank Katrina Krogh, MD, from essayreview.co.kr for editing a draft of this manuscript.

\section{CONFLICT OF INTEREST}

No potential conflict of interest relevant to this article was reported. 


\section{ORCID}

Won-Joong Kim, https://orcid.org/0000-0003-2046-8690

Hahck Soo Park, https://orcid.org/0000-0001-9782-8602

Min Ki Park, https://orcid.org/0000-0002-9320-5800

\section{REFERENCES}

1. Abejón D, Garcia-del-Valle S, Fuentes ML, Gómez-Arnau JI, Reig E, van Zundert J. Pulsed radiofrequency in lumbar radicular pain: clinical effects in various etiological groups. Pain Pract 2007; 7: 21-6.

2. Munglani R. The longer term effect of pulsed radiofrequency for neuropathic pain. Pain 1999; 80: 437-9.

3. Sluijter ME, Teixeira A, Van Duijn B. Comment on: Erdine S et al.; ultrastructural changes in axons following exposure to pulsed radiofrequency fields. Pain Pract 2010; 10: 262.

4. Kobayashi S, Yoshizawa H, Yamada S. Pathology of lumbar nerve root compression. part 2: morphological and immunohistochemical changes of dorsal root ganglion. J Orthop Res 2004; $22:$ 180-8.

5. Sugawara O, Atsuta Y, Iwahara T, Muramoto T, Watakabe $\mathrm{M}$, Takemitsu Y. The effects of mechanical compression and hypoxia on nerve root and dorsal root ganglia. an analysis of ectopic firing using an in vitro model. Spine (Phila Pa 1976) 1996; 21: 2089-94.

6. Shen J, Wang HY, Chen JY, Liang BL. Morphologic analysis of normal human lumbar dorsal root ganglion by 3D MR imaging. AJNR Am J Neuroradiol 2006; 27: 2098-103.

7. Lee IS, Kim SH, Lee JW, Hong SH, Choi JY, Kang HS, et al. Comparison of the temporary diagnostic relief of transforaminal epidural steroid injection approaches: conventional versus posterolateral technique. AJNR Am J Neuroradiol 2007; 28: 204-8.

8. Kim DH, Kim YC, Kim KH. Minimally invasive percutaneous spinal techniques. Philadelphia: Saunders Elsevier; 2011. pp 143-6.

9. Wolff AP, Groen GJ, Wilder-Smith OH. Influence of needle position on lumbar segmental nerve root block selectivity. Reg Anesth Pain Med 2006; 31: 523-30.
10. Gallizzi M, Gagnon C, Harden RN, Stanos S, Khan A. Medication quantification scale version III: internal validation of detriment weights using a chronic pain population. Pain Pract 2008; 8: 1-4.

11. Kim SJ, Park SJ, Yoon DM, Yoon KB, Kim SH. Predictors of the analgesic efficacy of pulsed radiofrequency treatment in patients with chronic lumbosacral radicular pain: a retrospective observational study. J Pain Res 2018; 11: 1223-30.

12. Das B, Conroy M, Moore D, Lysaght J, McCrory C. Human dorsal root ganglion pulsed radiofrequency treatment modulates cerebrospinal fluid lymphocytes and neuroinflammatory markers in chronic radicular pain. Brain Behav Immun 2018; 70: 157-65.

13. Howe JF, Loeser JD, Calvin WH. Mechanosensitivity of dorsal root ganglia and chronically injured axons: a physiological basis for the radicular pain of nerve root compression. Pain 1977; 3: 25-41.

14. Mikeladze G, Espinal R, Finnegan R, Routon J, Martin D. Pulsed radiofrequency application in treatment of chronic zygapophyseal joint pain. Spine J 2003; 3: 360-2.

15. Bogduk N. Pulsed radiofrequency. Pain Med 2006; 7: 396 407.

16. Chao SC, Lee HT, Kao TH, Yang MY, Tsuei YS, Shen CC, et al. Percutaneous pulsed radiofrequency in the treatment of cervical and lumbar radicular pain. Surg Neurol 2008; 70: 59-65.

17. Simopoulos TT, Kraemer J, Nagda JV, Aner M, Bajwa ZH. Response to pulsed and continuous radiofrequency lesioning of the dorsal root ganglion and segmental nerves in patients with chronic lumbar radicular pain. Pain Physician 2008; 11: 137-44.

18. Hasegawa T, Mikawa Y, Watanabe R, An HS. Morphometric analysis of the lumbosacral nerve roots and dorsal root ganglia by magnetic resonance imaging. Spine (Phila Pa 1976) 1996; 21: 1005-9.

19. Hasue M, Kunogi J, Konno S, Kikuchi S. Classification by position of dorsal root ganglia in the lumbosacral region. Spine (Phila Pa 1976) 1989; 14: 1261-4.

20. Hamanishi C, Tanaka S. Dorsal root ganglia in the lumbosacral region observed from the axial views of MRI. Spine (Phila Pa 1976) 1993; 18: 1753-6. 